\title{
Glueball as a bound state in the self-dual homogeneous vacuum gluon field
}

\author{
Ja. V. Burdanov, \\ Laboratory of Nuclear Problems, \\ Joint Institute for Nuclear Research, Dubna, Russia \\ G. V. Efimov, \\ Bogoliubov Laboratory of Theoretical Physics, \\ Joint Institute for Nuclear Research, Dubna, Russia
}

\begin{abstract}
Using a simple relativistic QFT model of scalar fields we demonstrate that the analytic confinement (propagator is an entire function in the complex $p^{2}$-plane) and the weak coupling constant lead to the Regge behaviour of the two-particle bound states.

In QCD we assume that the gluon vacuum is realized by the selfdual homogeneous classical field which is the solution of the YangMills equations. This assumption leads to analytical confinement of quarks and gluons. We extract the colorless $0^{++}$two-gluon state from the QCD generating functional in the one-gluon exchange approximation. The mass of this bound state is defined by the BetheSalpeter equation. The glueball mass varies in the region $1470 \mathrm{Mev} \leq$ $M_{0^{++}} \leq 1600 \mathrm{Mev}$ for $0.2 \leq \alpha_{s} \leq 0.5$ if the gluon condensate is $\frac{\alpha_{s}}{\pi}\left\langle G_{\mu \nu}^{a} G_{\mu \nu}^{a}\right\rangle=0.012(G e v)^{4}$.
\end{abstract}




\section{Introduction}

In this paper we shall calculate the mass of the scalar glueball $\mathrm{O}^{++}$. Before entering into a discussion, formulation and solution of this problem we would like to make some general remarks.

At the present time, QCD is considered to be an uniquely true theory of strong interactions, but there are different directions of its realization: lattice calculations, QCD sum rules, Nambu-Yona-Lasinio model plus or minus instantons, chiral perturbation theory, calculation based on the Wilson loop, bag and quasipotential models, models based on the analytic confinement, and maybe something else. It is useless to give references here because the literature is so numerous that it is practicaly impossible to list all the papers without missing some of them.

In our opinion, theoretical reasons for this variety consist in the following. From a general point of view the QFT methods can be used if the propagators

of particles and vertices, which describe interactions, are known and coupling constants are weak. Then perturbation calculations are valid and the BetheSalpeter equation is an adequate relativistic mathematical tool for computing bound states in QFT. The quantum electrodynamics, where the propagators and vertices are given and the coupling constant $\alpha=\frac{1}{137}$ is small, is the best example of this type of calculations.

However, in the case of QCD the situation is more difficult. Although the QCD coupling constant is considered to be relatively small $\alpha_{s}<1$, we can not be convinced that propagators of quarks and gluons have the standard Dirac and Klein-Gordon form for large distances, i.e., in the hadronization region. Unfortunately, a rigorous generally accepted analytic solution of this problem in QCD is still missing. Nevertheless, our hope is that a simple formalism similar to QED should exist in QCD, but a background form of these "basic bricks" is not yet found in QCD.

Another problem is that we do not have a self-consistent conventional theory of bound states in QFT at all and in QCD in particular, which can be applied in any cases like the Schrödinger equation is applied to any nonrelativistic quantum phenomena. Therefore different approaches with a doubtful basis are used to calculate bound states in relativistic cases. One can see that in most cases authors of different approaches strive to reduce the QFT bound state problem to a nonrelativistic Schrödinger equation with an appropriate potential. 
The generally accepted physical picture is that processes of hadronization take place at large distances where the confinement of quarks and gluons plays the main role. It should lead to the following statements. First, the free Dirac and Klein-Gordon equations for free particles are not adequate tools to describe quarks and gluons in this region. Second, currents, or vertices, which describe the connection of quarks and gluons with hadrons, cannot be local. In other words, only the knowledge of the vacuum QCD at these distances should dictate what equations have to describe quarks and gluons and nonlocal vertices. In addition, the QCD effective coupling constant $\alpha_{s}$ should be small enough in this hadronization region if we want to use the Bethe-Salpeter type equations.

We think that a successful guess about the structure of QCD vacuum at large distances can radically solve problems of confinement and hadronization.

In this paper, we want to show, using a simple model of scalar field, that in the frame work of QFT that analytic confinement of constituent particles (propagators are entire analytical functions in the momentum $p^{2}$-space) and weak coupling constant (the Bethe-Salpeter equation can be used) lead to the Regge spectrum of bound states.

Thus, if the QCD vacuum results in analytic confinement of quarks and gluons and the QCD coupling constant $\alpha_{s}$ is small, hadron bound states are expected to have the Regge type spectrum and we are able to calculate the masses of these states using the Bethe-Salpeter equation.

In this paper, we calculate the mass of a glueball with quantum numbers $0^{++}$. Glueballs are very interesting objects from both theoretical and experimental points of view. The experimental situation is not clear up to now (see, for example, [1, 2]), and we shall deal with it. However, the theoretical problem is quite intriguing: How can massless gluons be joined together into massive objects owing to the gluonic QCD self-interaction? It is evident that the solution of the glueball problem is directly connected with our understanding of nonperturbative Yang-Mills dynamics. Theoretical interest in glueballs arised just from the beginning of QCD and undoubtedly will continue until the structure of QCD vacuum is understood. All theoretical approaches (see, for example, the last papers [3, [1] where the previous publications are listed) are based on the understanding that the structure of QCD vacuum plays the decisive role in the solution of this problem, but all authors avoid to make any direct guesses about its explicit structure and take it into 
account in an indirect way only.

Our approach is based on the assumption that the QCD vacuum is realized by the self-dual homogeneous vacuum gluon field which is the classical solution of the Yang-Mills equations and in the one-loop approximation the QCD vacuum energy has minimum for nontrivial configurations of this field. This field leads to the analytic confinement of quarks and gluons, so that we could expect reasonable values for the glueball mass. This vacuum gluon field has been applied to quarkonia in papers [5, 6, 7]. We shall use the functional integral techniques to calculate mass spectrum of bound states (see 91). In this techniques the Bethe-Salpeter equation is written in a symmetrized form permitting variational calculations.

\section{The toy model}

In this section, we consider a simple quantum field model with confinement and demonstrate the features of possible bound states which can be interpreted as standard physical particles. We consider the scalar field $\varphi(x)$ with the Lagrangian in the Euclidean metrics

$$
\mathcal{L}(x)=-\frac{1}{2} \varphi(x) m^{2} e^{\frac{\square}{m^{2}}} \varphi(x)-g \varphi^{3}(x) .
$$

The equation for the free field $\phi(x)$ looks like

$$
m^{2} e^{\frac{\square}{m^{2}}} \varphi(x)=0, \quad \text { or } \quad m^{2} e^{-\frac{p^{2}}{m^{2}}} \tilde{\varphi}(p)=0
$$

and the solution $\phi(x) \equiv 0$ because the function $e^{-\frac{p^{2}}{m^{2}}} \neq 0$, i.e., it has no zeroes for any real or complex $p^{2}$. Exactly this property means analytic confinement. The field $\phi(x)$ can exist in the virtual state only, so that it can be called virton field (see [8]). In addition, one can say that the field $\phi(x)$ describes constituent particles.

The propagator

$$
\tilde{D}\left(p^{2}\right)=\frac{1}{m^{2}} e^{-\frac{p^{2}}{m^{2}}}, \quad D(x)=\int \frac{d k}{(2 \pi)^{4}} \tilde{D}(p) e^{i p x}=\frac{m^{2}}{(4 \pi)^{2}} e^{-\frac{m^{2}}{4} x^{2}} .
$$


is an entire analytical function in the complex $p^{2}$-plane. It guarantees the confinement of "particles" $\varphi(x)$ in each perturbation order over the dimensionless coupling constant

$$
\lambda=\left(\frac{3 g}{4 \pi m}\right)^{2} \leq 1
$$

The scale of the confinement region can be characterized by the value

$$
r_{c n f}^{2}=\frac{1}{\Lambda_{c n f}^{2}}=\frac{\int d x x^{2} D(x)}{\int d x D(x)}=\frac{8}{m^{2}} .
$$

The mechanism of arising of bound states can be described in the following way. Let us consider the partition function

$$
Z=\int \delta \varphi e^{\mathcal{L}[\varphi]}=\int \delta \varphi e^{-\frac{1}{2}\left(\varphi D^{-1} \varphi\right)+g \varphi^{3}} .
$$

The effective Lagrangian with the one-particle exchange reads

$$
\mathcal{L}_{\text {eff }}=-\frac{1}{2}\left(\varphi D^{-1} \varphi\right)+\frac{(3 g)^{2}}{2}\left(\varphi^{2} D \varphi^{2}\right)+\ldots
$$

The term $\left(\varphi^{2} D \varphi^{2}\right)$ can be transformed as

$$
\begin{aligned}
L_{2} & =\frac{(3 g)^{2}}{2} \iint d x_{1} d x_{2} \varphi^{2}\left(x_{1}\right) D\left(x_{1}-x_{2}\right) \varphi^{2}\left(x_{2}\right) \\
& =\frac{(3 g)^{2}}{2} \iint d x_{1} d x_{2} \varphi\left(x_{1}\right) \varphi\left(x_{2}\right) D\left(x_{1}-x_{2}\right) \varphi\left(x_{2}\right) \varphi\left(x_{1}\right) \\
& =\frac{(3 g)^{2}}{2} \int d x \int d y_{1} \sqrt{D\left(y_{1}\right)} \int d y_{2} \sqrt{D\left(y_{2}\right)} J\left(x, y_{1}\right) \delta\left(y_{1}-y_{2}\right) J^{+}\left(x, y_{2}\right),
\end{aligned}
$$

where $x_{1}=x+\frac{1}{2} y, \quad x_{2}=x-\frac{1}{2} y$ and

$$
\begin{gathered}
J(x, y)=\varphi\left(x+\frac{1}{2} y\right) \varphi\left(x-\frac{1}{2} y\right)=\varphi(x) e^{\frac{y}{2} \stackrel{\leftrightarrow}{\partial}} \varphi(x), \\
J^{+}(x, y)=J(x,-y) .
\end{gathered}
$$

Let us introduce an orthonormal system $\left\{U_{Q}(y)\right\}$, where $Q=(n, l,\{\mu\})$ are four-dimensional quantum numbers so that

$$
\int d y U_{Q}(y) U_{Q^{\prime}}(y)=\delta_{Q Q^{\prime}}, \quad \text { and } \quad \sum_{Q} U_{Q}(y) U_{Q}\left(y^{\prime}\right)=\delta\left(y-y^{\prime}\right) .
$$


Then

$$
\begin{aligned}
& L_{2}=\frac{(3 g)^{2}}{2} \int d x J_{Q}^{+}(x) \cdot J_{Q}(x), \\
& J_{Q}(x)=\varphi(x) V_{Q}(\stackrel{\leftrightarrow}{\partial}) \varphi(x), \quad J_{Q}^{+}(x)=\varphi(x) V_{Q}(-\stackrel{\leftrightarrow}{\partial}) \varphi(x), \\
& V_{Q}(\stackrel{\leftrightarrow}{\partial})=\int d y \sqrt{D(y)} U_{Q}(y) e^{\frac{y}{2}} \stackrel{\leftrightarrow}{\partial},
\end{aligned}
$$

and

$$
\begin{aligned}
& e^{\frac{(3 g)^{2}}{2}\left(\varphi^{2} D \varphi^{2}\right)}=\exp \left\{\frac{(3 g)^{2}}{2} \sum_{Q} \int d x J_{Q}^{+}(x) J_{Q}(x)\right\}, \\
& =\int d \sigma_{B B^{+}} \exp \left\{\frac{3 g}{\sqrt{2}} \sum_{Q} \int d x\left(B_{Q}^{+}(x) J_{Q}(x)+B_{Q}(x) J_{Q}^{+}(x)\right)\right\} \\
& =\int d \sigma_{B B^{+}} \exp \left\{\frac{3 g}{\sqrt{2}} \sum_{Q} \int d x \phi(x)\left[B_{Q}^{+}(x) V_{Q}(\stackrel{\leftrightarrow}{\partial})+B_{Q}(x) V_{Q}(-\stackrel{\leftrightarrow}{\partial})\right] \phi(x)\right\},
\end{aligned}
$$

where

$$
d \sigma_{B B^{+}}=\prod_{Q} \delta B_{Q} \delta B_{Q}^{+} e^{-\sum_{Q}\left(B_{Q}^{+} B_{Q}\right)} .
$$

We substitute this representation into the partition function $Z$ and after integration over $\varphi$ we get

$$
\begin{aligned}
Z & =\int \prod_{Q} \delta B_{Q} \delta B_{Q}^{+} e^{-\sum_{Q}\left(B_{Q}^{+} B_{Q}\right)-\frac{1}{2} \operatorname{Tr} \ln \left(1+3 g \sqrt{2}\left(B_{Q}^{+} V_{Q}+B_{Q} V_{Q}^{+}\right) D+\ldots\right)} \\
& =\int \prod_{Q} \delta B_{Q} \delta B_{Q}^{+} e^{-\sum_{Q Q^{\prime}}\left(B_{Q}^{+}\left[\delta_{Q Q^{\prime}}-\lambda \Pi_{Q Q^{\prime}}\right] B_{Q}\right)+O\left(B^{3}\right)}
\end{aligned}
$$

Here the polarization operator is

$$
\begin{aligned}
& \lambda \Pi_{Q Q^{\prime}}(z)=(3 g)^{2} \operatorname{Tr}\left(V_{Q}^{+} D V_{Q^{\prime}} D\right) \\
& =\iint d y_{1} d y_{2} U_{Q}\left(y_{1}\right) \lambda \Pi\left(z ; y_{1}, y_{2}\right) U_{Q^{\prime}}\left(y_{2}\right), \\
& \lambda \Pi\left(z ; y_{1}, y_{2}\right)=(3 g)^{2} \sqrt{D\left(y_{1}\right)} D\left(z+\frac{y_{1}-y_{2}}{2}\right) D\left(z-\frac{y_{1}-y_{2}}{2}\right) \sqrt{D\left(y_{2}\right)} .
\end{aligned}
$$


where $z=x_{1}-x_{2}$.

The Fourier transform looks like

$$
\lambda \tilde{\Pi}_{Q Q^{\prime}}(p)=\lambda \int d z e^{i p z} \Pi_{Q Q^{\prime}}(z)=\iint d y_{1} d y_{2} U_{Q}\left(y_{1}\right) S_{p}\left(y_{1}, y_{2}\right) U_{Q^{\prime}}\left(y_{2}\right),
$$

whith

$$
S_{p}\left(y_{1}, y_{2}\right)=\lambda \frac{m^{4}}{64 \pi^{2}} e^{-\frac{p^{2}}{2 m^{2}}} \cdot e^{-\frac{m^{2}}{4}\left(y_{1}^{2}-y_{1} y_{2}+y_{2}^{2}\right)} .
$$

The orthonormal system $\left\{U_{Q}(x)\right\}$ should diagonalize the kernel $S_{p}\left(y_{1}, y_{2}\right)$

$$
\lambda \tilde{\Pi}_{Q Q^{\prime}}(p)=\lambda_{Q}\left(p^{2}\right) \delta_{Q Q^{\prime}} .
$$

In other words, we have to find the spectrum and eigen functions of the problem

$$
S_{p} U_{Q}=\lambda_{Q}\left(p^{2}\right) U_{Q} \quad \text { or } \quad \int d y_{2} S_{p}\left(y_{1}, y_{2}\right) U_{Q}\left(y_{2}\right)=\lambda_{Q}\left(p^{2}\right) U_{Q}\left(y_{1}\right) .
$$

It should be stressed that this equation is nothing else but the Bethe-Salpeter equation in the one-boson exchange. In order to go to the standard form of the Bethe-Salpeter equation, we have to introduce new function $U_{Q}(y)=$ $\sqrt{D(y)} \Psi_{Q}(y)$ and go to the momentum representation (see, for example, [9]).

If we are able to do it, the partition function $Z$ in (7) looks like

$$
Z=\int \prod_{Q} \delta B_{Q} \delta B_{Q}^{+} e^{-\sum_{Q} \int d p\left(\tilde{B}_{Q}^{+}(p)\left[1-\lambda_{Q}\left(p^{2}\right)\right] \tilde{B}_{Q}(p)\right)+O\left(\tilde{B}^{3}\right)}
$$

and the equation

$$
1-\lambda_{Q}\left(-M_{Q}^{2}\right)=0
$$

defines the mass of the bound states with quantum numbers $Q$. Then

$$
1-\lambda_{Q}\left(p^{2}\right)=-\lambda_{Q}^{\prime}\left(-M_{Q}^{2}\right)\left(p^{2}+M_{Q}^{2}\right)+O\left(\left(p^{2}+M_{Q}^{2}\right)^{2}\right)
$$

and if

$$
-\lambda_{Q}^{\prime}\left(-M_{Q}^{2}\right)>0
$$


we can interpret the field $B_{Q}$ as a physical particle with quantum numbers $Q$ and mass $M_{Q}$.

The Bethe-Salpeter equation (9) can be solved and the eigen functions look like

$$
U_{Q}(y)=U_{n l\{\mu\}}(y)=C_{n l} T_{l\{\mu\}}(a y) L_{n}^{l+1}\left(a^{2} y^{2}\right) e^{-\frac{1}{2} a^{2} y^{2}}, \quad a^{2}=m^{2} \frac{\sqrt{3}}{2},
$$

Here, the angle polynomials $T_{l\{\mu\}}(y)=T_{l\left\{\mu_{1}, \ldots, \mu_{l}\right\}}(y)$ are normalized by

$$
\sum_{\{\mu\}} T_{l\{\mu\}}(x) T_{l\{\mu\}}(y)=C_{l}^{1}((x y))
$$

where $C_{l}^{1}(t)$ are the Gegenbauer polynomials. The eigenvalues are

$$
\lambda_{n l}\left(p^{2}\right)=\frac{\lambda e^{-\frac{p^{2}}{2 m^{2}}}}{(2+\sqrt{3})^{1+l+2 n}} .
$$

The equation (11) gives the spectrum

$$
M_{n l}^{2}=2 m^{2} \ln \left(\frac{\lambda_{c}}{\lambda}\right)+(l+2 n) \cdot 2 m^{2} \ln (2+\sqrt{3})
$$

where $\lambda_{c}=(2+\sqrt{3})^{2}=13.928 \ldots$. One can see that this spectrum has the pure Regge behaviour.

Now we can represent

$$
\begin{aligned}
1-\lambda_{Q}\left(p^{2}\right) & =1-\frac{\lambda}{\lambda_{c}} \cdot \frac{e^{\frac{M_{Q}^{2}}{2 m^{2}}}}{(2+\sqrt{3})^{l+2 n}} \cdot e^{-\frac{\left(p^{2}+M_{Q}^{2}\right)}{2 m^{2}}} \\
& =Z_{Q}\left(p^{2}+M_{Q}^{2}\right)+O\left(\left(p^{2}+M_{Q}^{2}\right)^{2}\right)
\end{aligned}
$$

with

$$
Z_{Q}=\frac{\lambda}{\lambda_{c}} \cdot \frac{e^{\frac{M_{Q}^{2}}{2 m^{2}}}}{(2+\sqrt{3})^{l+2 n}} \cdot \frac{1}{2 m^{2}} .
$$

Thus, the kinetic term in $Z(10)$ is

$$
\begin{aligned}
& \left(\tilde{B}_{Q}^{+}(p)\left[1-\lambda_{Q}\left(p^{2}\right)\right] \tilde{B}_{Q}(p)\right) \\
& =Z_{Q}\left(\tilde{B}_{Q}^{+}(p)\left[\left(p^{2}+M_{Q}^{2}\right)+O\left(\left(p^{2}+M_{Q}^{2}\right)^{2}\right)\right] \tilde{B}_{Q}(p)\right) .
\end{aligned}
$$


and after the renormalization

$$
\tilde{B}_{Q}(p) \rightarrow Z_{Q}^{-1 / 2} \tilde{B}_{Q}(p)
$$

becomes of the standard form.

In particular, the lowest scalar bound state $B_{0}$ has the mass

$$
M_{(00)}=m \sqrt{2 \ln \frac{\lambda_{c}}{\lambda}},
$$

For example, for $\lambda=.1$ we get

$$
M_{(00)}=3.142 \ldots \cdot m .
$$

Finally, we conclude:

- Bound states exist for small coupling constants

$$
\lambda<\lambda_{c} \approx 13
$$

and their masses grow when $\lambda \rightarrow 0$ as

$$
M_{Q} \sim m \sqrt{2 \ln \frac{\lambda_{c}}{\lambda} .}
$$

- Analytic confinement leads to the pure Regge spectrum for all bound states with quantum numbers $Q=(n l)$ and the slope of the Regge trajectories is defined by the scale of the confinement region $\Lambda_{c n f}^{2}$ and does not depend on the coupling constant $\lambda$.

- We can see that the size of the confinement region $r_{c n f}$ (ब4) excels remarkably the Compton length of all bound states

$$
l_{Q}=\frac{\hbar}{M_{Q} c} \ll r_{c n f} .
$$

It means that the physical particles which are described by the fields $B_{Q}(x)$ and all transformations with them take place in the confinement region induced by the constituent particles $\phi(x)$. 
- If $\lambda \leq .1$, i.e., we have the standard condition for perturbation calculations, then

$$
M \geq m \sqrt{2 \ln \left(10 \lambda_{c}\right)}=3.142 \ldots \cdot m .
$$

In other words, $M$ remarkably excels the "initial mass" $m$. If $\lambda$ is small enough, the bound state can be quite large.

This scheme will be applied in main features to find the glueball mass in QCD (see Table I). 


\begin{tabular}{|c|c|}
\hline Toy model & QCD \\
\hline 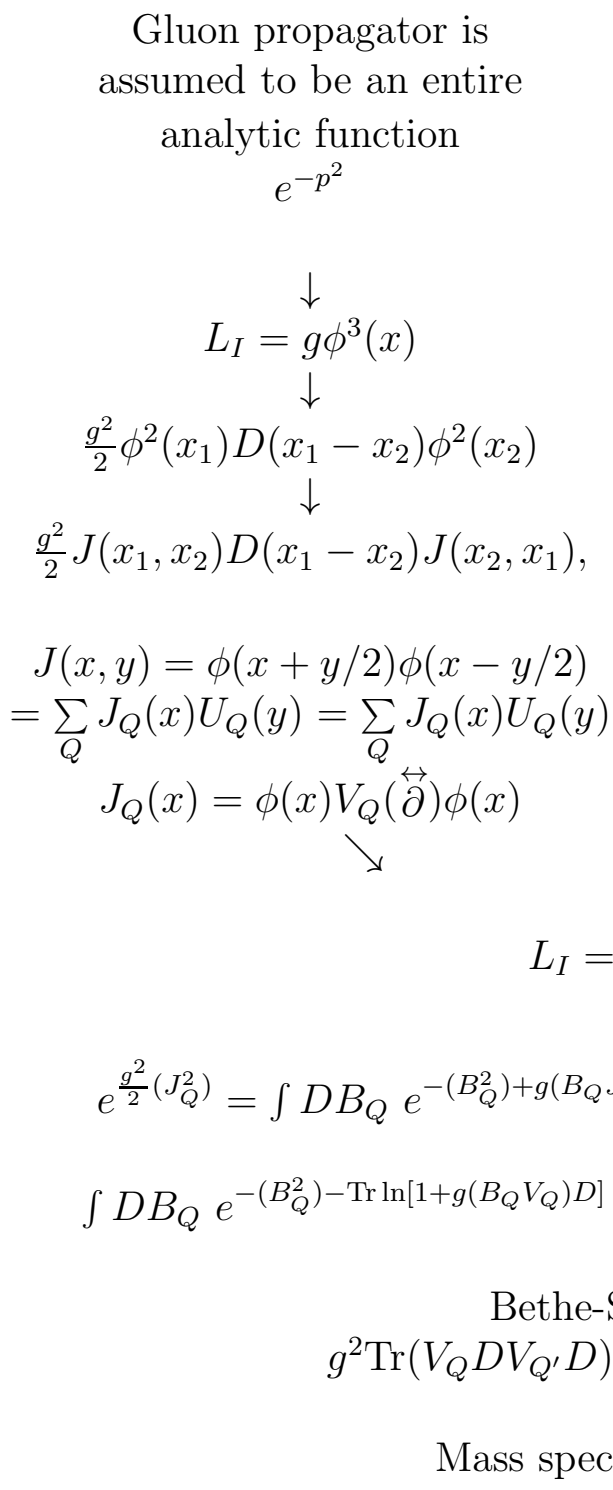 & 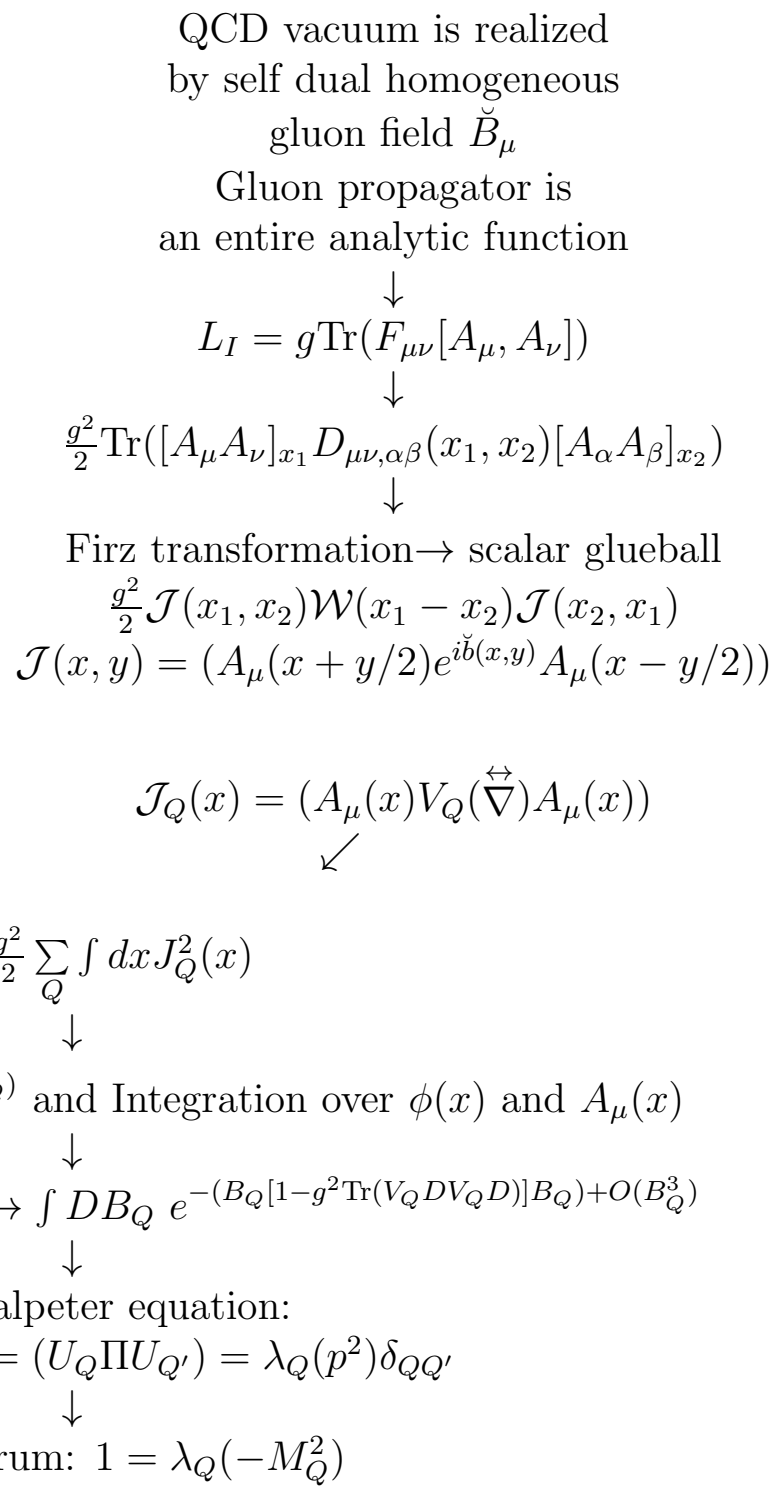 \\
\hline
\end{tabular}




\section{QCD Lagrangian in the self-dual gluon field}

Now let us go to QCD. The action has the form

$$
\begin{aligned}
& S=-\frac{1}{2} \int \mathrm{d}^{4} x \operatorname{Tr} \hat{G}_{\mu \nu} \hat{G}_{\mu \nu}, \\
& \hat{G}_{\mu \nu}=\partial_{\mu} \hat{A}_{\nu}-\partial_{\nu} \hat{A}_{\mu}-i g\left[\hat{A}_{\mu}, \hat{A}_{\nu}\right], \quad \hat{A}_{\mu}=t^{a} A_{\mu}^{a} .
\end{aligned}
$$

We suppose (see [5, 6, [7]) that the QCD vacuum is realized by a homogeneous (anti-)self-dual gluon field which has the form

$$
\begin{aligned}
& \hat{B}_{\mu}(x)=\hat{B}_{\mu \nu} x_{\nu}=\hat{n} B b_{\mu}(x)=\hat{n} B b_{\mu \nu} x_{\nu}, \\
& \hat{n}=t^{a} n^{a}, \quad \hat{n}^{+}=\hat{n}, \quad(n n)=n^{a} n^{a}=1, \\
& b_{\mu \rho} b_{\rho \nu}=-\delta_{\mu \nu}, \quad \tilde{b}_{\mu \nu}=\epsilon_{\mu \nu \alpha \beta} b_{\alpha \beta}= \pm b_{\mu \nu} .
\end{aligned}
$$

After substitution

$$
\hat{A}_{\mu}(x) \rightarrow \hat{A}_{\mu}(x)+\hat{B}_{\mu}(x)
$$

one has

$$
\hat{G}_{\mu \nu}=\hat{\mathcal{F}}_{\mu \nu}-i g\left[\hat{A}_{\mu}, \hat{A}_{\nu}\right]-2 \hat{B}_{\mu \nu}
$$

Here

$$
\hat{\mathcal{F}}_{\mu \nu}=\breve{\nabla}_{\mu} A_{\nu}-\breve{\nabla}_{\nu} A_{\mu}
$$

where we have used the notation

$$
\begin{array}{cc}
\breve{\nabla}_{\mu}^{a b}=\breve{\nabla}_{\mu}^{a b}(x)=\delta^{a b} \frac{\partial}{\partial x_{\mu}}-i \breve{\Omega}^{a b} b_{\mu}(x), \quad \breve{\nabla}_{\mu}=\frac{\partial}{\partial x_{\mu}}-i \breve{\Omega} b_{\mu \nu} x_{\nu}, \\
{\left[\breve{\nabla}_{\mu}, \breve{\nabla}_{\nu}\right]=2 i \breve{\Omega} b_{\mu \nu}, \quad \breve{\Omega}=g B \breve{n},} & \breve{\Omega}^{a b}=g B \breve{n}^{a b}, \\
\breve{n}^{a b}=-i f^{a b c} n^{c}, \quad \breve{n}^{+}=\breve{n}, \quad \breve{n}^{\top}=-\breve{n} .
\end{array}
$$

The QCD action (14) in the background field $B$ looks like

$$
\begin{aligned}
S & =-\frac{1}{2} \int \mathrm{d}^{4} x \operatorname{Tr}\left\{\hat{\mathcal{F}}_{\mu \nu} \hat{\mathcal{F}}_{\mu \nu}+4 i g \hat{B}_{\mu \nu}\left[\hat{A}_{\mu}, \hat{A}_{\nu}\right]\right. \\
& \left.-2 i g \hat{\mathcal{F}}_{\mu \nu}\left[\hat{A}_{\mu}, \hat{A}_{\nu}\right]+4 \hat{B}_{\mu \nu} \hat{B}_{\mu \nu}-g^{2}\left[\hat{A}_{\mu}, \hat{A}_{\nu}\right]^{2}\right\} .
\end{aligned}
$$


In order to fix a gauge, we should add to the action the gauge-fixed term

$$
S_{g f}=\int \mathrm{d}^{4} x \operatorname{Tr}\left(A_{\mu} \breve{\nabla}_{\mu}\right)\left(\breve{\nabla}_{\nu} A_{\nu}\right)=-\frac{1}{2} \int \mathrm{d}^{4} x\left(\breve{\nabla}_{\mu}^{a b} A_{\mu}^{b}\right)^{2} .
$$

After some transformations the QCD action is

$$
\begin{aligned}
S+S_{g f} & =\int \mathrm{d}^{4} x \operatorname{Tr}\left\{\left(A_{\mu} \breve{\nabla}_{\nu}\right)\left(\breve{\nabla}_{\nu} A_{\mu}\right)+8 i g \hat{A}_{\mu} \hat{B}_{\mu \nu} \hat{A}_{\nu}\right\} \\
& +\int \mathrm{d}^{4} x \operatorname{Tr}\left\{i g \hat{\mathcal{F}}_{\mu \nu}\left[\hat{A}_{\mu}, \hat{A}_{\nu}\right]+\frac{g^{2}}{2}\left[\hat{A}_{\mu}, \hat{A}_{\nu}\right]^{2}-2 \hat{B}_{\mu \nu} \hat{B}_{\mu \nu}\right\},
\end{aligned}
$$

The part of action quadratic over the quantum fields $A_{\mu}^{a}$ can be reduced to the form

$$
\int \mathrm{d}^{4} x \operatorname{Tr}\left[\left(A_{\mu} \breve{\nabla}_{\nu}\right)\left(\breve{\nabla}_{\nu} A_{\mu}\right)+8 i g \hat{A}_{\mu} \hat{B}_{\mu \nu} \hat{A}_{\nu}\right]=-\frac{1}{2} \int \mathrm{d}^{4} x A_{\mu}^{a}\left(G^{-1}\right)_{\mu \nu}^{a b} A_{\nu}^{b},
$$

where

$$
\left(G^{-1}\right)_{\mu \nu}^{a b}=\left[-\breve{\nabla}_{\rho}^{2} \delta_{\mu \nu}-4 i \breve{\Omega} b_{\mu \nu}\right]^{a b} .
$$

Finally, the QCD action reads

$$
\begin{aligned}
S & =S_{0}+S_{I}, \\
S_{0} & =-\frac{1}{2} \int d x A_{\mu}^{a}\left(G^{-1}\right)_{\mu \nu}^{a b} A_{\nu}^{b}=-\frac{1}{2}\left(A_{\mu} G_{\mu \nu}^{-1} A_{\nu}\right), \\
S_{I} & =\int \mathrm{d}^{4} x \operatorname{Tr}\left\{i g \hat{\mathcal{F}}_{\mu \nu}\left[\hat{A}_{\mu}, \hat{A}_{\nu}\right]+\frac{g^{2}}{2}\left[\hat{A}_{\mu}, \hat{A}_{\nu}\right]^{2}\right\} .
\end{aligned}
$$

\section{The Gluon Green function}

Let us find the gluon Green function corresponding to the operator (16). The $8 \times 8$ matrix $\breve{\Omega}$ for any vector $n^{a}$ has at least two zeroth eigenvalues. The choice of a vector $n^{a}$ requires special consideration and we plan to do it in further publications. In this paper, we choose the simplest case $n^{8}=$ 1, $n^{a}=0$ for $a=1, \ldots, 7$ which guarantees the quark confinement (see [5, 6, 7]). In this case, it is convenient to introduce the notion

$$
\breve{e}=\operatorname{diag}(1,1,1,0,0,0,0,1), \quad \breve{h}=\operatorname{diag}(0,0,0,1,1,1,1,0),
$$




$$
\begin{gathered}
\breve{e}+\breve{h}=\breve{I}=\operatorname{diag}(1,1,1,1,1,1,1,1), \\
\breve{\Omega}^{a b}=g B\left(-i f^{a b 8}\right)=\frac{\Lambda^{2}}{2} \breve{n}^{a b}, \quad g B=\Lambda^{2} \sqrt{3}, \quad \breve{n}^{2}=\breve{h} .
\end{gathered}
$$

In these notion the operator (16) can be written in the form

$$
\left(G^{-1}\right)_{\mu \nu}=-\partial_{\rho}^{2} P_{\mu \nu}^{(0)}+\left(-\breve{\nabla}_{\rho}^{2}+2 \Lambda^{2}\right) P_{\mu \nu}^{(+)}+\left(-\breve{\nabla}_{\rho}^{2}-2 \Lambda^{2}\right) P_{\mu \nu}^{(-)}
$$

where the projection operators are introduced

$$
P_{\mu \nu}^{(0)}=\breve{e} \delta_{\mu \nu}, \quad P_{\mu \nu}^{( \pm)}=\frac{1}{2}\left[\delta_{\mu \nu} \breve{h} \pm i \breve{n} b_{\mu \nu}\right], \quad\left(P_{\mu \nu}^{( \pm)}\right)^{\top}=P_{\nu \mu}^{( \pm)}
$$

Here the symbol " $*^{\top} "$ is connected with color indices.

The equation on the Green function looks like

$$
\begin{aligned}
& \left(G^{-1}\right)_{\mu \nu}^{a b} A_{\nu}^{b}(x)=\left[-\breve{\nabla}_{\rho}^{2} \delta_{\mu \nu}-4 i \breve{\Omega} b_{\mu \nu}\right]^{a b} A_{\nu}^{b}(x) \\
& =\left[-\partial_{\rho}^{2} P_{\mu \nu}^{(0)}+\left(-\breve{\nabla}_{\rho}^{2}+2 \Lambda^{2}\right) P_{\mu \nu}^{(+)}+\left(-\breve{\nabla}_{\rho}^{2}-2 \Lambda^{2}\right) P_{\mu \nu}^{(-)}\right] A_{\mu}(x)=J_{\mu}(x)
\end{aligned}
$$

Then, the Green function can be written in the form

$$
\begin{aligned}
G_{\mu \nu}(x, y)= & F_{\mu \nu}(x-y)+D_{\mu \nu}(x, y), \\
F_{\mu \nu}(x-y)= & F(Z) P_{\mu \nu}^{(0)}, \quad F(Z)=\frac{1}{-\partial_{\rho}^{2}} \delta(z), \\
D_{\mu \nu}(x, y)= & e^{i \breve{(}(x b y)} D_{\mu \nu}(z), \\
D_{\mu \nu}(z)= & D^{(+)}(Z) P_{\mu \nu}^{(+)}+D^{(-)}(Z) P_{\mu \nu}^{(-)}, \\
& D^{( \pm)}(Z)=\frac{1}{-\breve{\nabla}_{\rho}^{2}(z) \pm 2 \Lambda^{2}} \delta(z),
\end{aligned}
$$

where $z=x-y$ and $Z=\frac{\Lambda^{2} z^{2}}{4}$.

The free part of the Green function is

$$
F(Z)=\frac{1}{-\partial_{\rho}^{2}} \delta(z)=\left(\frac{\Lambda}{4 \pi}\right)^{2} \cdot \frac{1}{Z}
$$


The regular Green function $D^{(+)}$reads

$$
\begin{aligned}
D^{(+)}(Z) & =\frac{1}{-\breve{\nabla}_{\rho}^{2}(z)+2 \Lambda^{2}} \delta(z)=\left(\frac{\Lambda}{4 \pi}\right)^{2} \int_{1}^{\infty} d u \cdot \frac{u-1}{u+1} \cdot e^{-Z u} \\
& =\left(\frac{\Lambda}{4 \pi}\right)^{2}\left[\frac{e^{-Z}}{Z}+2 e^{Z} \operatorname{Ei}(-2 Z)\right] \rightarrow\left(\frac{\Lambda}{4 \pi}\right)^{2} \frac{e^{-Z}}{2 Z^{2}} \quad \text { for } \quad Z \rightarrow \infty
\end{aligned}
$$

It is convenient to represent

$$
D^{(+)}(Z)=\left(\frac{\Lambda}{4 \pi}\right)^{2} \int_{1}^{\infty} d \mathcal{D}_{c}^{(+)} e^{-Z c}
$$

The operator $-\breve{\nabla}_{\rho}^{2}(z)-2 \Lambda^{2}$ in the function $D^{(-)}(Z)$ contains the so called zeroth mode, i.e., there exists the eigen function $\Phi_{0}$ for which the eigen value equals 0

$$
\left[-\breve{\nabla}_{\rho}^{2}(z)-2 \Lambda^{2}\right] \Phi_{0}(z)=0, \quad \Phi_{0}(z)=C_{0} \cdot e^{-\frac{\Lambda^{2}}{4} z^{2}}
$$

In order to find the contribution of this zeroth mode to the Green function, we should solve the equation

$$
\left[-\breve{\nabla}_{\rho}^{2}(z)-2 \Lambda^{2}\right] \Psi_{0}(z)=J_{0} \cdot \Phi_{0}(z)=J_{0} \cdot e^{-\frac{\Lambda^{2}}{4} z^{2}} .
$$

This equation can be solved and the behaviour of this solution is

$$
\Psi_{0}(z) \sim e^{\frac{\Lambda^{2}}{4} z^{2}} \quad \text { for } \quad z^{2} \rightarrow \infty
$$

It means that this function does not belong to the space $\mathbf{L}^{2}$ and can not describe any physical state. Therefore, this function should be excluded from the set of physical states and the gluon Green function can not contain this contribution.

Thus, the Green function $D^{(-)}$without the zeroth mode looks like

$$
D^{(-)}(Z)=\operatorname{reg} \frac{1}{-\breve{\nabla}_{\rho}^{2}(z)-2 \Lambda^{2}} \delta(z)
$$


where reg means that the zeroth mode should be excluded. For this aim let us use the resolvent

$$
\begin{aligned}
R(\zeta) & =\frac{1}{-\breve{\nabla}_{\rho}^{2}(z)-2 \Lambda^{2}+\zeta} \delta(z)=\int_{0}^{\infty} d \alpha e^{\alpha \breve{\nabla}_{\rho}^{2}(z)+\left(2 \Lambda^{2}-\zeta\right) \alpha} \delta(z) \\
& =\int_{0}^{\infty} d \alpha e^{-\zeta \alpha}\left(\frac{\Lambda^{2} e^{\Lambda^{2} \alpha}}{4 \pi \sinh \left(\Lambda^{2} \alpha\right)}\right)^{2} \cdot e^{-\frac{z^{2} \Lambda^{2}}{4} \operatorname{coth}\left(\Lambda^{2} \alpha\right)} \\
& \rightarrow\left(\frac{\Lambda}{4 \pi}\right)^{2}\left\{\int_{0}^{\infty} d u\left[(\operatorname{coth}(u)+1)^{2} e^{-Z \operatorname{coth}(u)}-4 e^{-Z}\right]+\frac{4 \Lambda^{2}}{\zeta} e^{-Z}\right\}
\end{aligned}
$$

for $\zeta \rightarrow 0$.

Then, the Green function $D^{(-)}$is

$$
\begin{aligned}
D^{(-)}(Z) & =\left(\frac{\Lambda}{4 \pi}\right)^{2} \int_{1}^{\infty} \frac{d u}{u^{2}-1} \cdot\left[(u+1)^{2} e^{-Z u}-4 e^{-Z}\right] \\
& =\left(\frac{\Lambda}{4 \pi}\right)^{2}\left[\frac{1}{Z}-2 \mathbf{C}-2 \ln (2 Z)\right] \cdot e^{-Z} \\
& \rightarrow-\left(\frac{\Lambda}{4 \pi}\right)^{2} \ln (2 Z) e^{-Z} \quad \text { for } \quad Z \rightarrow \infty .
\end{aligned}
$$

Here, $\mathbf{C}=0.577215 \ldots$ is the Euler constant. We shall use the representation

$$
D^{(-)}(Z)=\left(\frac{\Lambda}{4 \pi}\right)^{2} \int_{1}^{\infty} d \mathcal{D}_{c}^{(-)} e^{-Z c} .
$$

Finally, the gluon Green function reads

$$
\begin{aligned}
& G_{\mu \nu}(x, y)=F(Z) \cdot P_{\mu \nu}^{(0)}+e^{i \check{\Omega}(x b y)} D_{\mu \nu}(Z), \\
& D_{\mu \nu}(Z)=D^{(+)}(Z) \cdot P_{\mu \nu}^{(+)}+D^{(-)}(Z) \cdot P_{\mu \nu}^{(-)} .
\end{aligned}
$$

\subsection{Confinement of gluons.}

Confinement problem: four color degrees of freedom are confined, but the other four color degrees of freedom are not confined. Our assumption is 
that the gluon vacuum has a domain structure $\mathbf{R}=\bigcup_{j} \Gamma_{j}$ with the scale of distances

$$
R_{\text {hadron }} \ll R_{\text {domain }} \ll R_{\text {exp }} .
$$

Here $R_{\text {hadron }} \sim \frac{1}{\Lambda}$ is a scale of regions where the strong quark-gluon-hadron interactions take place, $R_{\text {domain }}$ is a size of domains $\Gamma_{j}, R_{\text {exp }}$ is a macroscopic scale where hadrons are registered. Each domain is characterized by a direction of the color vector $n^{a}$ and this direction is supposed to be random.

Now we would like to give qualitative and semi-quantitative arguments that this structure of gluon vacuum can provide for the total gluon confinement if directions of the color vector $n^{a}$ are random in each domain. Let us consider for simplicity the case of group $S U(2)$. In this case we have the same problem. The $3 \times 3$ matrix $\breve{n}$ looks like

$$
\breve{n}^{a b}=-i \epsilon^{a b c} n^{c}, \quad\left(n^{c} n^{c}\right)=1, \quad(a, b, c=1,2,3)
$$

and the eigen values are $\lambda_{0}=0, \lambda_{ \pm}= \pm 1$. The qualitative behaviour of the gluon Green function is

$$
G^{a b}(z) \sim\left(e^{-Z \breve{n}}\right)^{a b} \sim \delta^{a b} e^{-Z}+n^{a} n^{b}\left(G_{0}-e^{-Z}\right)
$$

where $G_{0}$ is the free Green function. For $n=(0,0,1)$ we have

$$
G^{11}(z, n)=G^{22}(z)=e^{-Z}, \quad G^{33}(z)=G_{0},
$$

i.e., we have confinement for directions 1 and 2 and no confinement for direction 3 in the color space.

Now let us consider a gluon which moves in a direction through $N$ domains. It is clear that the terms with $e^{-Z}$ provide the confinement in each domain but the terms with $G_{0}$ permit gluons to travel at large distances. Let a gluon moves in a direction through $N$ domains. Its amplitude will be proportional to the factor

$$
A_{f i}^{a b}=n_{f}^{a}\left(n_{f} n_{N}\right)\left(n_{N} n_{N-1}\right) \cdot \ldots \cdot\left(n_{2} n_{1}\right)\left(n_{1} n_{i}\right) n_{i} .
$$

Here $n_{j}$ is the color vector of the domain $\Gamma_{j}$. We can consider $\left(n_{j} n_{j-1}\right)=$ $\cos \left(\theta_{j}\right)$ where $0 \leq \theta_{j} \leq \pi$ is assumed to be a random angle. Then, one gets

$$
\left|A_{f i}^{a b}\right| \leq \prod_{j=1}^{N}\left|\cos \left(\theta_{j}\right)\right|=\exp \left\{N \int_{0}^{\pi / 2} d \theta \ln \cos (\theta)\right\}=e^{-N c}
$$


where $c=1.0887 \ldots$. Thus, we see that a large number of domains can confine gluons.

\section{Two-gluon scalar colorless current}

The matrix element of the second order, which contains the scalar two-gluon current, looks like

$$
\begin{aligned}
L_{2} & =\frac{(i g)^{2}}{2} \iint d x d y \operatorname{Tr}(\left[\hat{A}_{\mu}(x), \hat{A}_{\nu}(x)\right] \overbrace{\left.\hat{\mathcal{F}}_{\mu \nu}(x)\right) \cdot \operatorname{Tr}\left(\hat{\mathcal{F}}_{\alpha \beta}\right.}(y)\left[\hat{A}_{\alpha}(y), \hat{A}_{\beta}(y)\right]) \\
& =-\frac{(2 i g)^{2}}{2} \iint d x d y T_{\mu \nu}^{a}(x)\left(-\breve{\nabla}_{\mu}^{a b}(x) \breve{\nabla}_{\alpha}^{s v}(y) G_{\nu \beta}^{b v}(x, y)\right) T_{\alpha \beta}^{s}(y) \\
& =2 g^{2} \iint d x d y T_{\mu \nu}^{a}(x)\left[e^{\frac{i}{2} \breve{b}(x, y)} \mathcal{D}_{\mu \alpha, \nu \beta}(x-y) e^{\frac{i}{2} \breve{b}(x, y)}\right]^{a s} T_{\alpha \beta}^{s}(y) \\
& =2 g^{2} \iint d x d y \mathcal{T}_{\mu \nu}^{a}(x)\left[e^{\frac{i}{2} \breve{b}(x, y)} \mathcal{S}_{\mu \alpha, \nu \beta}(x-y) e^{\frac{i}{2} \breve{b}(x, y)}\right]^{a s} \mathcal{T}_{\alpha \beta}^{s}(y),
\end{aligned}
$$

where

$$
T_{\mu \nu}^{a}(x)=\operatorname{Tr}\left(\left[\hat{A}_{\mu}(x), \hat{A}_{\nu}(x)\right] t^{a}\right), \quad \mathcal{T}_{\mu \nu}^{a}(x)=\operatorname{Tr}\left(\hat{A}_{\mu}(x) \hat{A}_{\nu}(x) t^{a}\right)
$$

and

$$
\begin{aligned}
& \mathcal{D}_{\mu \alpha, \nu \beta}^{a b}(z)=\left[\breve{\nabla}_{\mu}(z) \breve{\nabla}_{\alpha}^{\top}(z) D_{\nu \beta}(z)\right]^{a b} \\
& \mathcal{S}_{\mu \alpha, \nu \beta}^{a b}(z)=\mathcal{D}_{\mu \alpha, \nu \beta}^{a b}(z)+\mathcal{D}_{\nu \beta, \mu \alpha}^{a b}(z)-\mathcal{D}_{\nu \alpha, \mu \beta}^{a b}(z)-\mathcal{D}_{\mu \beta, \nu \alpha}^{a b}(z) .
\end{aligned}
$$
filed:

To obtain the function $\mathcal{D}_{\mu \alpha, \nu \beta}^{a b}(z)$ the following calculations must be ful-

$$
\begin{aligned}
& -\breve{\nabla}_{\mu}^{a b}(x) \breve{\nabla}_{\alpha}^{s v}(y) G_{\nu \beta}^{b v}(x, y)=-\breve{\nabla}_{\mu}^{a b}(x) G_{\nu \beta}^{b v}(x, y) \breve{\nabla}_{\alpha}^{\top v s}(y) \\
& =-\breve{\nabla}_{\mu}^{a b}(x) \breve{\nabla}_{\alpha}^{\top b v}(y) G_{\nu \beta}^{v s}(x, y)=-\breve{\nabla}_{\mu}^{a b}(x) \breve{\nabla}_{\alpha}^{\top b v}(y)\left[e^{i \breve{b}(x, y)} D_{\nu \beta}(x-y)\right]^{v s} \\
& =-\left[e^{i b(x, y)} \breve{\nabla}_{\mu}(x-y) \breve{\nabla}_{\alpha}^{\top}(y-x) D_{\nu \beta}(x-y)\right]^{a s} \\
& =e^{i \breve{b}(x, y)} \breve{\nabla}_{\mu}(z) \breve{\nabla}_{\alpha}^{\top}(z) D_{\nu \beta}(z)=e^{\frac{i}{2} \breve{b}(x, y)} \mathcal{D}_{\mu \alpha, \nu \beta}(z) e^{\frac{i}{2} \breve{b}(x, y)} .
\end{aligned}
$$

Using the identity

$$
t_{i j}^{a}\left(e^{\frac{i}{2} \breve{b}(x, y)}\right)^{a b}=\left(e^{\frac{i}{2} \hat{b}(x, y)} t^{b} e^{-\frac{i}{2} \hat{b}(x, y)}\right)_{i j}
$$


one can obtain

$$
\begin{aligned}
& \operatorname{Tr}\left(\hat{A}_{\mu}(x) \hat{A}_{\nu}(x) t^{a}\right)\left(e^{\frac{i}{2} \breve{b}(x, y)}\right)^{a b}=\operatorname{Tr}\left(\hat{A}_{\mu}(x, y) \hat{A}_{\nu}(x, y) t^{b}\right) \\
& \left(e^{\frac{i}{2} \breve{b}(x, y)}\right)^{r s} \operatorname{Tr}\left(t^{s} \hat{A}_{\alpha}(y) \hat{A}_{\beta}(y)\right)=\operatorname{Tr}\left(t^{r} \hat{A}_{\alpha}(y, x) \hat{A}_{\beta}(y, x)\right)
\end{aligned}
$$

where the bilocal gluon fields have the form

$$
\hat{A}_{\mu}(x, y)=e^{-\frac{i}{2} \hat{b}(x, y)} \hat{A}_{\mu}(x) e^{\frac{i}{2} \hat{b}(x, y)}, \quad \hat{A}_{\alpha}(y, x)=e^{-\frac{i}{2} \hat{b}(y, x)} \hat{A}_{\alpha}(y) e^{\frac{i}{2} \hat{b}(y, x)}
$$

So we can rewrite $L_{2}$ as the follows

$$
L_{2}=2 g^{2} \iint d x d y \operatorname{Tr}\left(\hat{A}_{\mu}(x, y) \hat{A}_{\nu}(x, y) t^{a}\right) \mathcal{S}_{\mu \alpha, \nu \beta}^{a s}(x-y) \operatorname{Tr}\left(t^{s} \hat{A}_{\alpha}(y, x) \hat{A}_{\beta}(y, x)\right) .
$$

In order to extract the scalar colorless two-gluon state, the following standard steps should be done:

1. Using the Fierz transformation for the $t^{a}$-matrices

$$
t_{i j}^{a} t_{k n}^{b}=\frac{1}{18} \delta^{a b} \delta^{j k} \delta^{i n}+\ldots
$$

we extract the four-gluon color singlet

$$
\begin{aligned}
& \operatorname{Tr}\left(\hat{A}_{\mu}(x, y) \hat{A}_{\nu}(x, y) t^{a}\right) \mathcal{S}_{\mu \alpha, \nu \beta}^{a s}(x-y) \operatorname{Tr}\left(t^{s} \hat{A}_{\alpha}(y, x) \hat{A}_{\beta}(y, x)\right) \\
& =\frac{1}{18} \operatorname{Tr}\left(\hat{A}_{\mu}(x, y) \hat{A}_{\nu}(x, y) \hat{A}_{\alpha}(y, x) \hat{A}_{\beta}(y, x)\right) \mathcal{S}_{\mu \alpha, \nu \beta}^{s s}(x-y)+\ldots
\end{aligned}
$$

2. Next we extract the two-gluon color singlet and octet:

$$
\begin{aligned}
& \operatorname{Tr}\left(\hat{A}_{\mu}(x, y) \hat{A}_{\nu}(x, y) \hat{A}_{\alpha}(y, x) \hat{A}_{\beta}(y, x)\right) \\
& =\left(\hat{A}_{\nu}(x, y) \hat{A}_{\alpha}(y, x)\right)_{i j}\left(\hat{A}_{\beta}(y, x) \hat{A}_{\mu}(x, y)\right)_{j i} \\
& =\frac{1}{3} \operatorname{Tr}\left(\hat{A}_{\nu}(x, y) \hat{A}_{\alpha}(y, x)\right) \cdot \operatorname{Tr}\left(\hat{A}_{\beta}(y, x) \hat{A}_{\mu}(x, y)\right) \\
& +2 \operatorname{Tr}\left(t^{a} \hat{A}_{\nu}(x, y) \hat{A}_{\alpha}(y, x)\right) \cdot \operatorname{Tr}\left(t^{a} \hat{A}_{\beta}(y, x) \hat{A}_{\mu}(x, y)\right)
\end{aligned}
$$

3. The two-gluon color singlet can be represented

$$
\mathcal{J}_{\mu \alpha}(x, y)=\frac{\delta_{\mu \alpha}}{8} \mathcal{J}(x, y)+\mathcal{J}_{\mu \alpha}^{(2)}(x, y)
$$


where $\mathcal{J}(x, y)$ and $\mathcal{J}_{\mu \alpha}^{(2)}(x, y)$ describes spins zero and two respectively. For spin zero we have

$$
\begin{aligned}
\mathcal{J}(x, y) & =2 \operatorname{Tr}\left(\hat{A}_{\sigma}(x, y) \hat{A}_{\sigma}(y, x)\right)=2 \operatorname{Tr}\left(\hat{A}_{\sigma}(x) e^{i \hat{b}(x, y)} \hat{A}_{\sigma}(y) e^{i \hat{b}(y, x)}\right) \\
& =A_{\sigma}^{a}(x)\left(e^{i \breve{b}(x, y)}\right)^{a b} A_{\sigma}^{b}(y)=\left(A_{\sigma}(x) e^{i \breve{b}(x, y)} A_{\sigma}(y)\right) .
\end{aligned}
$$

4. The effective "potential" connecting two scalar currents is

$$
\begin{aligned}
\mathcal{V}(z) & =\frac{1}{2} \mathcal{S}_{\mu \nu, \nu \mu}^{a a}(z)=\mathcal{D}_{\mu \nu, \nu \mu}^{a a}(z)-\mathcal{D}_{\mu \mu, \nu \nu}^{a a}(z) \\
& =\operatorname{Tr}\left[\breve{\nabla}_{\mu}(z) \breve{\nabla}_{\nu}^{\top}(z) D_{\nu \mu}(z)-\breve{\nabla}_{\mu}(z) \breve{\nabla}_{\mu}^{\top}(z) D_{\nu \nu}(z)\right] .
\end{aligned}
$$

Finally, we get

$$
L_{2}=\frac{g^{2}}{864} \iint d x_{1} d x_{2} \mathcal{J}\left(x_{1}, x_{2}\right) \mathcal{V}\left(x_{1}-x_{2}\right) \mathcal{J}\left(x_{2}, x_{1}\right)
$$

\subsection{The gluon-gluon "potential".}

The gluon-gluon "potential" $\mathcal{V}(z)$ can be calculated

$$
\begin{aligned}
\mathcal{V}(z) & =\operatorname{Tr}\left[\breve{\nabla}_{\mu}(z) \breve{\nabla}_{\nu}^{\top}(z) D_{\nu \mu}(z)-\breve{\nabla}_{\mu}(z) \breve{\nabla}_{\mu}^{\top}(z) D_{\nu \nu}(z)\right] . \\
& =\operatorname{Tr}\left[\breve{\nabla}_{\mu}(z) P_{\mu \nu}^{(+)} \breve{\nabla}_{\nu}^{\top}(z)-\breve{\nabla}_{\mu}(z) \breve{\nabla}_{\mu}^{\top}(z) P_{\nu \nu}^{(+)}\right] D^{(+)}(Z) \\
& +\operatorname{Tr}\left[\breve{\nabla}_{\mu}(z) P_{\mu \nu}^{(-)} \breve{\nabla}_{\nu}^{\top}(z)-\breve{\nabla}_{\mu}(z) \breve{\nabla}_{\mu}^{\top}(z) P_{\nu \nu}^{(-)}\right] D^{(-)}(Z)
\end{aligned}
$$

One can get

$$
\begin{aligned}
& {\left[\breve{\nabla}_{\mu}(z) P_{\mu \nu}^{( \pm)} \breve{\nabla}_{\nu}^{\top}(z) D^{( \pm)}(Z)-\breve{\nabla}_{\mu}(z) \breve{\nabla}_{\mu}^{\top}(z) P_{\nu \nu}^{( \pm)} D^{( \pm)}(Z)\right] } \\
= & \breve{h}\left\{\frac{2}{3}\left[-\breve{\nabla}^{2}(z) \pm 2 \Lambda^{2}\right]+\frac{\Lambda^{2}}{2}\left[\mp 4-6 Z \pm 2 Z \frac{d}{d Z}\right] D^{( \pm)}(Z)\right\} .
\end{aligned}
$$

The first term gives $\delta(z)$ and this part of our potential describes the local interaction which leads to the standard renormalization and contributions to the highest perturbation orders. Thus, the potential that is responsible for the glueball bound state reads

$$
\begin{aligned}
\mathcal{W}(z) & =4 \Lambda^{2}\left\{\left[-2-3 Z+Z \frac{d}{d Z}\right] D^{(+)}(Z)+\left[2-3 Z-Z \frac{d}{d Z}\right] D^{(-)}(Z)\right\} \\
& =\left(\frac{\Lambda^{2}}{2 \pi}\right)^{2} \int_{1}^{\infty} d \mathcal{W}_{a} e^{-Z a}
\end{aligned}
$$


It is usefull to introduce the representation

$$
\sqrt{\mathcal{W}(Z)}=\frac{\Lambda^{2}}{2 \pi} \int_{1}^{\infty} d \mathcal{U}_{a} e^{-Z a} .
$$

\section{Nonlocal Currents and Bosonization}

Now let us go to $L_{2}$ in (30) and consider the scalar current $J(x, y)$, which in the center of mass system of two gluons $\left(x_{1}=x+\frac{y}{2}, x_{2}=x-\frac{y}{2}\right)$ becomes

$$
\begin{aligned}
\mathcal{J}(x, y) & =\left(A_{\sigma}\left(x+\frac{y}{2}\right) e^{i \breve{b}\left(x+\frac{y}{2}, x-\frac{y}{2}\right)} A_{\sigma}\left(x-\frac{y}{2}\right)\right) \\
& =\left(A_{\sigma}(x) e^{\frac{y}{2} \stackrel{\leftrightarrow}{\nabla}(x)} A_{\sigma}(x)\right)
\end{aligned}
$$

where

$$
\begin{aligned}
& \overleftrightarrow{\nabla}_{\mu}(x)=\overleftarrow{\nabla}_{\mu}(x)-\vec{\nabla}_{\mu}(x), \\
& \vec{\nabla}_{\mu}(x)=\vec{\partial}_{\mu}-i \breve{\Omega} b_{\mu \nu} x_{\nu}, \quad \overleftarrow{\nabla}_{\mu}(x)=\overleftarrow{\partial}_{\mu}+i \breve{\Omega} b_{\mu \nu} x_{\nu}
\end{aligned}
$$

Then

$$
\begin{aligned}
L_{2} & =\frac{g^{2}}{864} \iint d x d y \mathcal{J}(x, y) \mathcal{W}(y) \mathcal{J}(x,-y) \\
& =\frac{g^{2}}{864} \int d x \iint d y_{1} d y_{2} \sqrt{\mathcal{W}\left(y_{1}\right)} \mathcal{J}\left(x, y_{1}\right) \delta^{4}\left(y_{1}-y_{2}\right) \sqrt{\mathcal{W}\left(y_{2}\right)} \mathcal{J}\left(x,-y_{2}\right)
\end{aligned}
$$

Let us introduce an orthonormal system of functions $\left\{U_{Q}(y)\right\}$

$$
\sum_{Q} U_{Q}\left(y_{1}\right) U_{Q}\left(y_{2}\right)=\delta\left(y_{1}-y_{2}\right), \quad \int d y U_{Q}(y) U_{Q^{\prime}}(y)=\delta_{Q Q^{\prime}}
$$

where $Q$ are quantum numbers. We obtain

$$
L_{2}=\frac{g^{2}}{864} \sum_{Q} \int d x \mathcal{J}_{Q}(x) \cdot \mathcal{J}_{Q}^{+}(x)
$$

where

$$
\begin{aligned}
& \mathcal{J}_{Q}(x)=\left(A_{\sigma}(x) V_{Q}(\stackrel{\leftrightarrow}{\nabla}(x)) A_{\sigma}(x)\right) \\
& V_{Q}(\overleftrightarrow{\nabla}(x))=\int d y \sqrt{\mathcal{W}(y)} U_{Q}(y) e^{\frac{y}{2} \stackrel{\leftrightarrow}{\nabla}(x)}
\end{aligned}
$$


We are interested in the scalar glueball which is described by the current $\mathcal{J}_{0}$, i.e., with the quantum numbers $Q=0$. The part of $L_{2}$ with these quantum numbers looks like

$$
\begin{aligned}
& L_{2}=\frac{g_{1}^{2}}{4} \int d x \mathcal{J}_{0}^{2}(x), \quad g_{1}^{2}=\frac{g^{2}}{216} \\
& \mathcal{J}_{0}(x)=\left(A_{\sigma}(x) V_{0}(\stackrel{\leftrightarrow}{\nabla}(x)) A_{\sigma}(x)\right)
\end{aligned}
$$

The scalar gluon current consists of the confined and non-confined gluon modes

$$
\mathcal{J}=\mathcal{J}^{(f)}+\mathcal{J}^{(c)}=\left(A_{\mu}^{(f)} V_{0} A_{\mu}^{(f)}\right)+\left(A_{\mu}^{(c)} V_{0} A_{\mu}^{(c)}\right)
$$

It should be stressed that this division is $S U(3)$ invariant because the eigen numbers of the matrix $\breve{n}$ are invariants of $S U(3)$ group transformations. Therefore,

$$
\left(A_{\mu} G_{\mu \nu}^{-1} A_{\nu}\right)=\left(A_{\mu}^{(c)} G_{\mu \nu}^{-1} A_{\nu}^{(c)}(x)\right)+\left(A_{\mu}^{(f)} G_{\mu \nu}^{-1} A_{\nu}^{(f)}(x)\right) .
$$

Our main assumption is that only confined modes lead to a bound state, but local modes lead to standard renormalizations and contributions to next perturbation orders. It means that the part of the partition function, which is responsible for the glueball formation, reads

$$
\begin{aligned}
Z & =\int D A^{(c)} e^{-\frac{1}{2}\left(A^{(c)} D^{-1} A^{(c)}\right)+\frac{g_{1}^{2}}{4}\left(\mathcal{J}_{0}^{(c)} \mathcal{J}_{0}^{(c)}\right)} \\
& =\int D \varphi e^{-\frac{1}{2}(\varphi \varphi)} \int D A^{(c)} e^{-\frac{1}{2}\left(A^{(c)} D^{-1} A^{(c)}\right)+\frac{g_{1}}{\sqrt{2}}\left(\mathcal{J}_{0}^{(c)} \varphi\right)} \\
& =\int D \varphi e^{-\frac{1}{2}(\varphi \varphi)} \int D A^{(c)} e^{-\frac{1}{2}\left(A^{(c)} D^{-1} A_{\nu}^{(c)}\right)+\frac{g_{1}}{\sqrt{2}}\left(A^{(c)} \varphi V_{0} A^{(c)}\right)} \\
& =\int D \varphi e^{-\frac{1}{2}(\varphi \varphi)-\frac{1}{2} \operatorname{Tr} \ln \left(1-\sqrt{2} g_{1} \varphi V_{0} D\right)} .
\end{aligned}
$$

Expanding the logarithm, we extract the quadratic part over $\varphi(x)$ in the form

$$
Z=\int D \varphi e^{-\frac{1}{2}\left(\varphi \mathcal{H}^{-1} \varphi\right)+O\left(\phi^{3}\right)} .
$$

where

$$
\mathcal{H}^{-1}\left(x_{1}-x_{2}\right)=\delta\left(x_{1}-x_{2}\right)-g_{1}^{2} \Pi\left(x_{1}-x_{2}\right),
$$




$$
\Pi\left(x_{1}-x_{2}\right)=\operatorname{Tr}\left\{V_{0}\left(\overleftrightarrow{\nabla}_{x_{1}}\right) D\left(x_{1}, x_{2}\right) V_{0}\left(\overleftrightarrow{\nabla}_{x_{2}}\right) D\left(x_{2}, x_{1}\right)\right\}
$$

The polarization operator $\Pi(z)$ can be written as follows $\left(z=x_{1}-x_{2}\right)$

$$
\Pi(z)=\iint d y_{1} d y_{2} \sqrt{\mathcal{W}\left(y_{1}\right)} U_{0}\left(y_{1}\right) \Pi\left(z \mid y_{1}, y_{2}\right) \sqrt{\mathcal{W}\left(y_{2}\right)} U_{0}\left(y_{2}\right)
$$

where

$$
\begin{aligned}
& \Pi\left(z \mid y_{1}, y_{2}\right) \\
& =\operatorname{Tr}\left\{e^{\frac{y_{1}}{2} \stackrel{\leftrightarrow}{\nabla}\left(x_{1}\right)} e^{i \breve{\Omega}\left(x_{1} b x_{2}\right)} D_{\alpha \beta}\left(x_{1}-x_{2}\right) e^{\frac{y_{2}}{2} \stackrel{\leftrightarrow}{\nabla}\left(x_{2}\right)} e^{i \breve{\Omega}\left(x_{2} b x_{1}\right)} D_{\beta \alpha}^{\top}\left(x_{2}-x_{1}\right)\right\} \\
& =\operatorname{Tr}\left\{e^{\frac{y_{1}}{2} \stackrel{\leftrightarrow}{\nabla}(z)} D_{\alpha \beta}(z) e^{\frac{y_{2}}{2} \stackrel{\leftrightarrow}{\nabla}(z)} D_{\beta \alpha}^{\top}(z)\right\} \\
& =\operatorname{Tr}\left\{e^{i \breve{\Omega}\left(\left(y_{1}-y_{2}\right) b z\right)} D_{\alpha \beta}\left(z-\frac{y_{1}+y_{2}}{2}\right) D_{\beta \alpha}^{\top}\left(-z-\frac{y_{1}+y_{2}}{2}\right)\right\} \\
& =2 \operatorname{Tr}\left[\breve{h} e^{i \breve{\Omega}\left(\left(y_{1}-y_{2}\right) b z\right)} \sum_{j= \pm} D^{(j)}\left(Z_{(+)}\right) D^{(j)}\left(Z_{(-)}\right)\right]
\end{aligned}
$$

with

$$
Z_{( \pm)}=\frac{\Lambda^{2}}{4}\left(z \pm \frac{y_{1}+y_{2}}{2}\right)^{2}
$$

\subsection{Polarization operator}

We do not know the eigen function $U_{0}(y)$; so we shall use the variation method with the test function

$$
U_{0}(y, \gamma)=\frac{\Lambda^{2}}{2 \pi} \cdot e^{-\gamma Y}, \quad Y=\frac{\Lambda^{2}}{4} y^{2},
$$

where $\gamma$ is a variation parameter. Our point of view is that this function should be a quite good approximation for the ground state of the BetheSalpeter equation.

The Fourier transfrmation of the polarization operator looks like

$$
\begin{aligned}
& \tilde{\Pi}\left(p^{2}, \gamma\right)=\int d z e^{i p z} \Pi(z)=\frac{1}{\pi^{2}}\left(\frac{\Lambda^{2}}{4 \pi}\right)^{6} \cdot R\left(p^{2}, \gamma\right) \\
& R\left(p^{2}, \gamma\right)=\gamma^{2} \iint_{1}^{\infty} d \mathcal{U}_{a_{1}} d \mathcal{U}_{a_{2}} \sum_{j= \pm} \iint_{1}^{\infty} d \mathcal{D}_{c_{1}}^{(j)} d \mathcal{D}_{c_{2}}^{(j)} I\left(a_{1}, a_{2}, c_{1}+\gamma, c_{2}+\gamma ; p^{2}\right)
\end{aligned}
$$




$$
\begin{aligned}
& I\left(a_{1}, a_{2}, c_{1}, c_{2} ; p^{2}\right)=\operatorname{Tr} 2 \breve{h} \int d z \iint d y_{1} d y_{2} \\
& \cdot e^{i p z-a_{1} \frac{y_{1}^{2} \Lambda^{2}}{4}-a_{2} \frac{y_{2}^{2} \Lambda^{2}}{4}-c_{1} \frac{\Lambda^{2}}{4}\left(z-\frac{y_{1}+y_{2}}{2}\right)^{2}-c_{2} \frac{\Lambda^{2}}{4}\left(z+\frac{y_{1}+y_{2}}{2}\right)^{2}+i \breve{\Omega}\left(\left(y_{1}-y_{2}\right) b z\right)} \\
& =\left(\frac{4 \pi}{\Lambda^{2}}\right)^{6} \cdot \frac{8}{\left[K\left(a_{1}, a_{2}, c_{1}, c_{2}\right)\right]^{2}} \cdot e^{-\frac{p^{2}}{\Lambda^{2}} \cdot \frac{k\left(a_{1}, a_{2}, c_{1}, c_{2}\right)}{K\left(a_{1}, a_{2}, c_{1}, c_{2}\right)}}, \\
& k\left(a_{1}, a_{2}, c_{1}, c_{2}\right)=a_{1} a_{2}+\frac{1}{4}\left(a_{1}+a_{2}\right)\left(c_{1}+c_{2}\right), \\
& K\left(a_{1}, a_{2}, c_{1}, c_{2}\right)=\left(a_{1}+a_{2}\right)\left(1+c_{1} c_{2}\right)+\left(c_{1}+c_{2}\right)\left(1+a_{1} a_{2}\right) .
\end{aligned}
$$

Finally, we have

$$
\begin{aligned}
& g_{1}^{2} \tilde{\Pi}\left(p^{2}, \gamma\right) \\
& =\frac{\alpha_{s}}{\pi} \cdot \frac{4 \gamma^{2}}{27} \iint_{1}^{\infty} d \mathcal{U}_{a_{1}} d \mathcal{U}_{a_{2}} \sum_{j= \pm} \iint_{1}^{\infty} d \mathcal{D}_{c_{1}}^{(j)} d \mathcal{D}_{c_{2}}^{(j)} \cdot \frac{\exp \left\{-\frac{p^{2}}{\Lambda^{2}} \cdot \frac{k\left(a_{1}, a_{2}, c_{1}+\gamma, c_{2}+\gamma\right)}{K\left(a_{1}, a_{2}, c_{1}+\gamma, c_{2}+\gamma\right)}\right\}}{\left[K\left(a_{1}, a_{2}, c_{1}+\gamma, c_{2}+\gamma\right)\right]^{2}}
\end{aligned}
$$

where $\alpha_{s}=\frac{g^{2}}{4 \pi}$. Next step is to calculate

$$
\max _{\gamma} g_{1}^{2} \tilde{\Pi}\left(-\frac{M^{2}}{\Lambda^{2}}, \gamma\right)=\frac{\alpha_{s}}{\pi} \cdot \tilde{\Pi}\left(\frac{M^{2}}{\Lambda^{2}}\right)
$$

and the mass of scalar glueball is defined by the equation

$$
\frac{\alpha_{s}}{\pi} \cdot \tilde{\Pi}\left(\frac{M^{2}}{\Lambda^{2}}\right)=1
$$

The results of calculations are given in the Table. 


\begin{tabular}{|c|c|c|}
\hline$\frac{M}{\Lambda}$ & $\alpha_{s}$ & $M$ \\
& & $(\Lambda=446 \mathrm{Mev})$ \\
\hline 3.0 & 1.5120 & 1337 \\
3.1 & 1.0609 & 1381 \\
3.2 & 0.7369 & 1426 \\
3.3 & 0.5067 & 1470 \\
& & \\
3.4 & 0.3450 & 1515 \\
3.5 & 0.2325 & 1560 \\
3.6 & 0.1551 & 1604 \\
& & \\
3.7 & 0.1024 & 1649 \\
3.8 & 0.0669 & 1694 \\
3.9 & 0.0432 & 1738 \\
4.0 & 0.0277 & 1782 \\
\hline
\end{tabular}

The gluon condensate

$$
\begin{aligned}
& \left\langle G_{\mu \nu}^{a} G_{\mu \nu}^{a}\right\rangle=B_{\mu \nu}^{a} B_{\mu \nu}^{a}=n^{a} n^{a} B^{2} b_{\mu \nu} b_{\mu \nu}=4 B^{2}, \\
& \Lambda^{4}=\frac{1}{3} g^{2} B^{2}=\frac{\pi^{2}}{3} \cdot \frac{g^{2}}{4 \pi^{2}} \cdot 4 B^{2}=\frac{\pi^{2}}{3} \cdot \frac{\alpha_{s}}{\pi} \cdot\left\langle G_{\mu \nu}^{a} G_{\mu \nu}^{a}\right\rangle=\frac{\pi^{2}}{3}\left\langle\mathcal{O}_{G}\right\rangle \\
& \left\langle\mathcal{O}_{G}\right\rangle=.012 x_{G}(G e v)^{4}, \\
& \Lambda=446 x_{G}^{.25} M e v,
\end{aligned}
$$

where $x_{G} \approx 1$ (see, for example, [10]). The values of $\alpha_{s}\left(\Lambda^{2}\right)$ are given in [1].

\subsection{Conclusion}

In the conclusion we would like to list the main points of our approach.

- Analytic confinement plus weak coupling lead to the Regge spectrum of bound states. 
- Gluon self-dual vacuum field $\hat{B}_{\mu}(x)$ leads to full quark confinement and partial gluon confinement.

- The field $\hat{B}_{\mu}(x)$ has directions in color and configuration spaces; so the domain structure of the whole space $\mathbf{R}^{4}$ with appropriate averaging over these directions is needed to provide full gluon confinement and relativistic covariance.

- Gluon propagator in the field $\hat{B}_{\mu}(x)$ has the zeroth modes, which are not the Goldstone degrees of freedom. We exclude them, because they lead to a nonphysical growth of the propagator in the $x$-space for $x \rightarrow \infty$.

- Numerical results are quite reasonable and with the results of [5, 6, 7] give hope that our main assumption about $\hat{B}_{\mu}(x)$ contains "a grain of truth".

\subsection{Appendix}

We use the following method of approximations. Let a positive function

$$
f(t)=\int_{1}^{\infty} d \sigma_{u} e^{-t u}
$$

be given, where $d \sigma_{u}$ is a positive measure.

Our aim is to get the approximation

$$
f(t) \approx f_{a}(t)=\sum_{j=1}^{n} A_{j} e^{-a_{j} t}
$$

on the interval $0 \leq t_{i} \leq t \leq t_{f} \leq \infty$.

Our procedure is the following. Let us choose $n$ points

$$
\left\{t_{1}, t_{2}, \ldots, t_{n}\right\}, \quad t_{j} \in\left[t_{i}, t_{f}\right]
$$

and calculate

$$
a_{j}=-\frac{f^{\prime}\left(t_{j}\right)}{f\left(t_{j}\right)}, \quad(j=1, \ldots, n) .
$$


Then, we solve the system of equations

$$
f\left(t_{i}\right)=\sum_{j=1}^{n} A_{j} e^{-a_{j} t_{i}}, \quad(i=1, \ldots, n)
$$

so that we get the numbers

$$
\left\{A_{1}, \ldots, A_{n}\right\}
$$

and we have the approximation

$$
f(t)=\int_{1}^{\infty} d \sigma_{u} e^{-t u} \approx f_{a}(t)=\sum_{j=1}^{n} A_{j} e^{-a_{j} t} .
$$

The accuracy of this approximation can be evaluated by

$$
\delta=\frac{\left\|f-f_{a}\right\|}{\|f\|}, \quad\|f\|=\int_{t_{i}}^{t_{f}} d t|f(t)|
$$

The accuracy depends on a particular choice of the points $\left\{t_{1}, \ldots, t_{n}\right\}$.

In our case, we have

\begin{tabular}{|c|c|c|c|c|c|c|}
\hline & \multicolumn{2}{|c|}{$\sqrt{\mathcal{W}(t)}$} & \multicolumn{2}{|c|}{$D^{(+)}(t)$} & \multicolumn{2}{|c|}{$D^{(-)}(t)$} \\
\hline$t_{j}$ & $.04, .4,1,2.2,4,7,10$ & $.01, .05, .2, .4,1.2$ & $.01, .05, .1, .3, .5, .9,3$ \\
\hline $\mathrm{j}$ & $A_{j}$ & $a_{j}$ & $A_{j}$ & $a_{j}$ & $A_{j}$ & $a_{j}$ \\
\hline & & & & & & \\
1 & 7.009 & 3.341 & 179.4 & 106.3 & 175.2 & 96.62 \\
2 & 0.485 & 2.227 & 29.42 & 24.04 & 36.16 & 19.76 \\
3 & -8.234 & 0.959 & 6.334 & 7.607 & 152.7 & 10.94 \\
4 & -1.532 & 0.002 & 0.281 & 4.592 & 27.77 & 6.554 \\
5 & 55.497 & 0.144 & 1.031 & 2.365 & -171.8 & 10.46 \\
6 & -257.1 & 0.197 & & & 3.971 & 1.836 \\
7 & 208.3 & 0.215 & \multicolumn{3}{|c|}{0.027} \\
\end{tabular}




\section{References}

[1] F.E.Close. "Glueballs: A central mystery", hep-ph/0006288 26.06.00;

[2] J.Ellis, H.Fujii, D.Kharzeev. "Scalar Glueball-Quarkonium Mixing and Structure of QCD Vacuum". hep-ph/9909322 10.09.99;

[3] A.B.Kaidalov, Yu.A.Simonov, "Glueball masses and Pomeron trajectory in nonperturbative QCD approach", hep-ph/9912434 (2000),

[4] H.Forkel, "Scalar Gluonium and Instantons", hep-hp/0005004 (2000);

[5] G V Efimov, S.N. Nedelko, Phys. Rev. D51 (1995) 174; Eur. Phys. J. C1 (1998) 343;

[6] Ja. V Burdanov, G V Efimov, S.N. Nedelko, S.A. Solunin, Phys. Rev. D54 (1996) 4483;

[7] G V Efimov, A.C. Kalloniatis, S.N. Nedelko, Phys. Rev. D59 (1999) 014026;

[8] G.V.Efimov, M.A.Ivanov, "The Quark Confinement Model of Hadrons", IOP Publishing Ltd, London, 1993;

[9] G.V. Efimov, "Bound states in QFT, scalar fields." hep-ph/9907483 (1999);

[10] M.A.Shifman, ITEP Lectures on Particle Physics and Field Theory, Vol I, World Scientific Lecture Notes, World Scientific- Vol 62, Singapure, 1999 ;

[11] I.Hinchliffe. "The QCD Coupling Constant". hep-ph/0004186 19.04.00. 